\title{
Recomendación para el tratamiento con hipotermia en recién nacidos con encefalopatía hipóxico-isquémica
}

\author{
Grupo de Trabajo Hipotermia Terapéutica \\ Comité de Estudios Feto-Neonatales (CEFEN)
}

Coordinadora: Dra. Silvia Fernández Jonusas (Hospital Italiano de Buenos Aires)

Daniela Satragno (Hospital de Niños Ricardo Gutiérrez)

Elba López Turconi (Sanatorio de Los Arcos)

Gustavo Goldsmit (Hospital Garrahan)

Cecilia Rubio (Hospital Garrahan)

Guillermo Colantonio (Clínica y Maternidad Suizo Argentina)

Viviana Robledo (Sanatorio Otamendi y Miroli)

Juan Pablo Berazategui (Hospital Universitario Austral)

Mariana Valera (Hospital Fernández)

Nora Balanian (Maternidad Ramón Sardá)

Teresa Mazzuchelli (Hospital Garrahan)

Luis Ahumada (Hospital de la Misericordia de Córdoba)

http: / / dx.doi.org/10.5546/ aap.2017.s38

Resumen ejecutivo publicado en Arch Argent Pediatr 2017;115(3):300-303

Cómo citar: Grupo de Trabajo Hipotermia Terapéutica,
Comité de Estudios Feto-Neonatales (CEFEN).
Recomendación para el tratamiento con hipotermia en
recién nacidos con encefalopatía hipóxico-isquémica.
Arch Argent Pediatr 2017;115 Supl 3:s38-s52.

Correspondencia:

Dra. Silvia Fernández Jonusas:

silvia.fernandez@

hospitalitaliano.org.ar

Financiamiento:

Ninguno.

Conflicto de intereses: Ninguno que declarar.

Recibido: 20-11-2016 Aceptado: 29-11-2016

\section{RESUMEN}

La encefalopatía hipóxico-isquémica es una causa importante de daño neurológico agudo en el recién nacido. Desde fines de la década del 2000, existe creciente evidencia científica de que el enfriamiento corporal total o de la cabeza es una terapia eficaz para su tratamiento. Esta terapia debe emplearse con los criterios de inclusión que utilizaron los diferentes estudios aleatorizados controlados y se debe realizar en centros de alta complejidad que permitan resolver las potenciales complicaciones. El énfasis debería estar puesto en la detección precoz de estos niños, debido a la estrecha ventana terapéutica y a que, muchas veces, deben ser referidos a centros de mayor complejidad. Implementar esta terapéutica permitirá reducir la mortalidad y la discapacidad mayor en los sobrevivientes.

Con el objeto de unificar criterios de atención de estos pacientes, el Comité de Estudios Feto-neonatales ha elaborado la siguiente recomendación.

\section{INTRODUCCIÓN}

La encefalopatía hipóxicoisquémica $(\mathrm{EHI})$ es una causa importante de daño neurológico agudo en el recién nacido ( $\mathrm{RN}$ ). Desde hace un tiempo, existe creciente evidencia científica de que el enfriamiento corporal total o de la cabeza es una terapia eficaz para su tratamiento, ya que disminuye la morbimortalidad. Esta terapia debe utilizarse con los criterios de inclusión que fueron publicados en los estudios de investigación. La hipotermia terapéutica se debe realizar en centros de alta complejidad que permitan resolver las potenciales complicaciones. El énfasis debería estar puesto en la detección precoz de estos niños, debido a la estrecha ventana terapéutica y a que, muchas veces, deben ser referidos a estos centros, lo que permitirá reducir la mortalidad y la discapacidad mayor en los sobrevivientes.

Con el objeto de unificar criterios de atención de estos pacientes, el Comité de Estudios Feto-neonatales (CEFEN) ha elaborado la siguiente recomendación. 


\section{ÍNDICE}

Introducción

Pág. S38

Información general

Pág. 540

Fisiopatología de la asfixia

Pág. 540

Mecanismos de acción de la hipotermia

Pág. S41

Identificación de los recién nacidos con diagnóstico de

encefalopatía hipóxico-isquémica candidatos a recibir tratamiento con hipotermia

Pág. 541

$\mathrm{Al}$ nacer

Pág. S41

Criterios de exclusión

Pág. 542

Manejo en la sala de partos

Pág. 542

Traslado

Pág. 542

Traslado desde la sala de partos

Pág. 542

Traslado hacia otro centro de atención neonatal

Pág. 542

Mantenimiento de la temperatura durante el período de estabilización y transporte

Pág. 543

Manejo del paciente durante la hipotermia

Pág. 543

Medidas generales

Pág. 543

Realización de controles de laboratorio

Pág. S45

Pasos iniciales del tratamiento

Pág. 546

Fases

Fase de enfriamiento

Pág. 546

Fase de mantenimiento

Pág. S46

Fase de recalentamiento

Pág. 546

Pág. 546

Modos de tratamiento

Pág. S46

Hipotermia pasiva

Pág. 547

Hipotermia corporal total

Pág. S47

Hipotermia cerebral selectiva

Pág. 547

Tratamiento farmacológico

Pág. 547

Pág. 547

Monitoreo de la función cerebral

Pág. 547

Monitoreo por electroencefalograma de amplitud integrada

Pág. 548

¿Cómo se evalúan los registros del electroencefalograma de amplitud integrada?

Pág. S49

Actividad convulsiva

Pág. 549

Pág. S50

Estudio del sistema nervioso central con imágenes

Pág. S51

Potenciales auditivos evocados automatizados

Pág. S51

Nuevos tratamientos coadyuvantes

Pág. S51

Seguimiento del paciente

Pág. S51

Bibliografía 


\section{INFORMACIÓN GENERAL}

Durante el siglo xx, la EHI ha estado huérfana de intervenciones terapéuticas específicas dirigidas a prevenir o aminorar el daño cerebral asociado a la agresión perinatal. Los neonatos con EHI moderada y grave (EHI significativa) constituyen, por su riesgo de muerte o de secuelas neurológicas, un reto terapéutico. En el año 2006, la Academia Americana de Pediatría (AAP) determinó que esta patología era "una de las frustraciones clínicas no resueltas de la medicina neonatal contemporánea". ${ }^{1}$ Las implicancias médicas, sociales y legales que asocia esta devastadora entidad son importantes y condicionan un problema sociosanitario relevante. ${ }^{2,3}$

La EHI es una causa importante de daño neurológico agudo en el $\mathrm{RN}$ a término y pretérmino tardío. Su incidencia en países desarrollados es, aproximadamente, de entre 1 y 3 casos x $1000 \mathrm{RN}$ vivos de edad gestacional $\geq 36$ semanas.

Varios ensayos clínicos indican que la reducción de la temperatura cerebral entre $3{ }^{\circ} \mathrm{C}$ y $4{ }^{\circ} \mathrm{C}$, mediante un enfriamiento corporal total o selectivo de la cabeza, constituye una intervención eficaz y segura para reducir la mortalidad y la discapacidad mayor en los sobrevivientes. ${ }^{4-7}$

Esta intervención terapéutica es más efectiva cuanto más temprana sea aplicada y dentro de una ventana terapéutica comprendida dentro de las seis horas de vida. Una vez alcanzada la temperatura objetivo de $33{ }^{\circ} \mathrm{C}-34{ }^{\circ} \mathrm{C}$, esta hipotermia moderada debe ser mantenida durante 72 horas, tras lo cual se realiza un recalentamiento lento.

La eficacia y seguridad observada en los ensayos clínicos ha sido ratificada en, al menos, cuatro metaanálisis (Edwards et al., ${ }^{8}$ Schulzke et al., ${ }^{9}$ Shah et al., ${ }^{10}$ y Jacobs et al. ${ }^{11}$ ). Todos concluyen lo siguiente sobre esta intervención:

a) Es eficaz para reducir la prevalencia de muerte-discapacidad asociada a la EHI moderada-grave.

b) El número de neonatos con EHI que precisan ser tratados para prevenir un caso de muerte o discapacidad está en un rango de 6 a 9 pacientes.

c) Es una intervención terapéutica segura, sin riesgo relevante para el bebé siempre que se aplique en unidades de nivel III y siguiendo protocolos estrictos de enfriamiento y recalentamiento.

d) Esta reducción en el daño se sostiene en el tiempo evaluado en el seguimiento a largo plazo.

La EHI constituye una urgencia neurológica, debido a la necesidad de iniciar la hipotermia dentro de las 6 hs de vida. ${ }^{12,13}$

En la última actualización de reanimación neonatal International Liaisson Committee on Resuscitation (ILCOR) 2015, se explicitó que podía considerarse el uso de hipotermia terapéutica utilizando protocolos claramente definidos, similares a los utilizados en los ensayos clínicos en centros con capacidad para proporcionar una atención multidisciplinaria y para realizar el seguimiento de los pacientes. ${ }^{14,15}$

Este documento desarrollado por el Grupo de Trabajo Hipotermia Terapéutica CEFEN de la Sociedad Argentina de Pediatría (SAP) tiene como objetivo ofrecer recomendaciones que sirvan en nuestro entorno para lograr estandarizar el tratamiento con hipotermia en la EHI.

\section{Fisiopatología de la asfixia}

La EHI se produce como consecuencia de la privación de oxígeno al cerebro por hipoxemia arterial, por isquemia cerebral o por la concurrencia de ambas situaciones.

El daño en la EHI es un proceso complejo, que comienza con el insulto hipóxico y continúa durante el período de recuperación. ${ }^{16}$ Este proceso consta de varias fases: inicialmente y de forma inmediata tras la agresión, tiene lugar una despolarización celular hipóxica y un fracaso energético primario; es la denominada fase primaria del daño. En esta, un número determinado de neuronas pueden morir por necrosis celular durante la situación aguda. Durante la reperfusión, tiene lugar una recuperación parcial del metabolismo oxidativo del cerebro (fase latente). Luego de esta, el metabolismo puede deteriorarse nuevamente (fase de fracaso energético secundario). ${ }^{17,18}$ Es en esta última fase cuando se produce la muerte por necrosis y por apoptosis de los elementos celulares neurales.

El fracaso energético secundario se caracteriza por alterar los gradientes iónicos transmembrana, liberar neuroaminoácidos al compartimento extracelular y activar una serie de reacciones bioquímicas y moleculares en cascada que extienden y agravan el daño durante las horas siguientes y conducen a una amplia, aunque no uniforme, distribución de muerte celular necrótica y apoptótica. En modelos animales de agresión hipóxico-isquémica, esta fase se caracteriza por 
la aparición de convulsiones, edema citotóxico, acumulación de neuroaminoácidos excitatorios, génesis de radicales libres de oxígeno, alteración de la función mitocondrial y activación de mecanismos apoptóticos. ${ }^{19,20}$

La EHI está presente desde el nacimiento, y no existe un período libre de sintomatología clínica. El perfil neurológico evolutivo en el curso de los primeros días o semanas permite diferenciar la EHI perinatal de una encefalopatía neonatal de otro origen. El curso temporal es de gran valor para establecer más certeramente el pronóstico. En general, en la EHI leve y moderada, el cuadro clínico comienza a mejorar de manera progresiva después de las 72 horas de vida. En la EHI grave, el RN está estuporoso o en coma, intensamente hipotónico y puede presentar convulsiones sutiles o clónicas multifocales. Entre las 24 y las 72 horas de vida, parece agudizarse el deterioro de la capacidad para despertar $y$, con frecuencia, aparece disfunción del tronco encefálico y algunos RN presentan signos de hipertensión endocraneana. En la evolución natural, es en este período cuando el neonato habitualmente fallece. Los que sobreviven experimentan una progresiva mejoría en la vigilia, el tono muscular cambia progresivamente de la hipotonía inicial a distonía o hipertonía extensora y puede aparecer una combinación de parálisis bulbar y pseudobulbar que determina problemas en la alimentación.

\section{Mecanismos de acción de la hipotermia}

- Reduce el metabolismo cerebral, previene el edema.

- Disminuye la utilización de energía.

- Disminuye/suprime la acumulación de aminoácidos citotóxicos y óxido nítrico.

- Inhibe el factor de activación plaquetaria y la cascada inflamatoria.
- Suprime la actividad de los radicales libres.

- Atenúa la falla de energía secundaria.

- Inhibe la apoptosis neuronal.

- Reduce la extensión de la lesión cerebral.

Identificación de los recién nacidos con diagnóstico de encefalopatía hipóxico-isquémica candidatos a recibir tratamiento con hipotermia

- Edad gestacional $\geq 35$ semanas.

- Edad posnatal $\leq 6 \mathrm{~h}$ de vida sin haber iniciado ningún tipo de control de la temperatura central.

- Identificación de un evento centinela.

Para asignar el término EHI perinatal, es necesaria la ocurrencia de una alteración neurológica aguda producida por falta de adecuado transporte de oxígeno durante el curso del trabajo de parto o parto.

La existencia de un patrón de frecuencia cardíaca fetal no tranquilizador $\mathrm{u}$ otros eventos, como desprendimiento de placenta, prolapso de cordón, rotura uterina, distocia de parto, embolia de líquido amniótico, hemorragia materna, bradicardia fetal grave y sostenida, trauma o paro cardiorrespiratorio y período expulsivo prolongado, son considerados eventos centinela. ${ }^{21}$

\section{Al nacer}

- $\mathrm{pH}$ de cordón o pH de sangre del bebé en la primera hora de vida $\leq 7 \mathrm{y} / \mathrm{o}$ déficit de base $\geq-12$.

- Puntaje de Apgar a los 10 min $\leq 5$.

- Reanimación con ventilación a presión positiva durante más de $10 \mathrm{~min}$.

- Evidencia de encefalopatía de moderada a grave definida como convulsiones clínicas o la presencia de 1 signo en, al menos, 3 de las 6 categorías evaluadas en la clasificación de Sarnat y Sarnat (Tabla 1).

TABLA 1. Clasificación de la encefalopatía hipóxico-isquémica de Sarnat y Sarnat

\begin{tabular}{lll}
\hline & Grado de encefalopatía & (Sarnat y Sarnat) \\
\hline Categoría & Moderada & Grave \\
\hline Nivel de conciencia & Letargo. & Estupor, coma. \\
Postura & Disminuida. & Sin actividad. \\
& Flexión distal. Extensión total. & Brazos extendidos y rotados internamente. \\
Tono & Hipotónico. & Piernas extendidas con pies en flexión plantar forzada. \\
Reflejos primitivos & Reflejo de succión débil. & Fláccido. \\
& Reflejo de Moro incompleto. & Ausencia de reflejo de succión y reflejo de Moro ausente. \\
Sistema autonómico & Pupilas contraídas. Bradicardia. & Variables, desiguales, no reactivas a la luz, dilatadas. \\
& Respiración periódica. & Frecuencia cardíaca variable. Apnea. \\
\hline
\end{tabular}




\section{Criterios de exclusión}

- Falta de evidencia de EHI o asfixia perinatal.

- Pacientes con peso al nacer (PN) menor de 1800 gramos.

- Trastornos de la coagulación sanguínea que no se corrigen con la administración de plaquetas, plasma o crioprecipitados.

- Pacientes cuyo estado general o condición clínica es muy grave y se acuerda con los padres la futilidad del tratamiento.

NOTA: Las anomalías congénitas mayores únicas o múltiples y las anomalías cromosómicas deberán ser consideradas de manera particular por el equipo tratante.

\section{Manejo en la sala de partos}

De acuerdo con el programa de reanimación neonatal, se recomienda lo siguiente:

- Comienzo de la reanimación con aire a mbiente ( $21 \%$ de oxígeno). Cuando la reanimación se inicia con aire ambiente, está demostrado que los bebés comienzan el primer llanto más rápidamente que el grupo reanimado con oxígeno al $100 \%$ (1,2 $\pm 0,6$ vs. $1,7 \pm 0,5$ minutos). Los bebés establecen la respiración efectiva en menos tiempo $(4,6 \pm 0,7$ vs. 7,5 $\pm 1,8$ minutos) y muestran menores hallazgos bioquímicos de estrés oxidativo. ${ }^{22}$

- Aunque no hay estudios clínicos sobre el uso de oxígeno durante la reanimación cardiopulmonar (RCP), el grupo de redacción de las guías de RCP neonatal de la SAP continúa respaldando el uso de oxígeno al $100 \%$ cuando se realizan compresiones torácicas (RCP avanzado). Es razonable reducir gradualmente la concentración de oxígeno en cuanto se recupera la frecuencia cardíaca. ${ }^{15}$

- La utilización de saturometría de pulso es de suma importancia para conocer el requerimiento de oxígeno que precisa el paciente. Tanto la hipoxia como la hiperoxia ejercen efectos deletéreos sobre el sistema nervioso central (SNC). ${ }^{15}$

- Colocación de un monitor electrocardiográfico para la evaluación de la frecuencia cardíaca.

- Las drogas (adrenalina, naloxona) deben ser preparadas y administradas según se establece en el Manual de Reanimación Cardiopulmonar Neonatal de la Sociedad Argentina de Pediatría. Se deben evitar expansiones bruscas de la volemia y administración de bicarbonato y /o calcio en forma de bolo.
- Cuando se inicia reanimación en pacientes de 35 o más semanas, se sugiere apagar la fuente de calor y colocar el sensor de temperatura central al paciente. Una vez establecida la ventilación y una frecuencia cardíaca adecuada, se debe mantener un estrecho monitoreo de esta con valores de temperatura central en $35^{\circ} \mathrm{C}$ hasta decidir si el paciente debe ser incluido en un programa de hipotermia terapéutica. Si el paciente se encuentra en un hospital de baja complejidad, se deberá iniciar el trámite de derivación lo antes posible. Se debe evitar hipertermia, temperatura $\left(\mathrm{T}^{\circ}\right)>37^{\circ} \mathrm{C}$ e hipotermia, temperatura por debajo de $33^{\circ} \mathrm{C}$.

- Se debe realizar un estado ácido-base (EAB) de cordón umbilical o de sangre arterial lo más cercano al nacimiento y antes de la hora de vida del paciente. Si el EAB se realiza más cerca de la hora de vida, el $\mathrm{pH}$ puede ser modificado a expensas de la disminución de dióxido de carbono $\left(\mathrm{CO}_{2}\right)$, por lo tanto, se sugiere tomar en cuenta otras variables, como exceso de bases y ácido láctico.

- Si el paciente restablece la circulación y permanece con ventilación asistida, se debe evitar la hiperventilación, que podría conducir a hipocapnia, vasoconstricción cerebral y disminución del flujo sanguíneo cerebral.

- Se debe realizar un chequeo de la glucemia y, si es necesario, colocar infusión de glucosa a $6 \mathrm{mg} / \mathrm{k} /$ minuto para evitar la hipoglucemia posterior a la reanimación.

- Se debe informar a los padres la condición clínica del bebé.

- Se debe informar a la Unidad de Cuidados Intensivos Neonatales (UCIN) la situación clínica del paciente.

\section{TRASLADO}

\section{Traslado desde la sala de partos}

El traslado desde la sala de partos debiera ser realizado en las mismas condiciones y con el mismo monitoreo y cuidados con el que se realizan las transferencias de los pacientes críticos. Este cuidado incluye saturometría continua y monitoreo electrocardiográfico, incubadora de transporte, con especial atención en mantener la temperatura corporal entre 34 y 35 grados.

Traslado hacia otro centro de atención neonatal

Debido a que el enfriamiento es más eficaz cuanto antes se inicie, el enfriamiento pasivo 
debe empezar tan pronto como sea posible. En condiciones ideales, deberá comenzar en el mismo lugar del nacimiento. ${ }^{23}$

\section{Mantenimiento de la temperatura durante el} período de estabilización y transporte ${ }^{24}$

- Se debe controlar y registrar la temperatura rectal cada 15 minutos.

- Objetivo: mantener el rango de temperatura rectal entre $33{ }^{\circ} \mathrm{C}$ y $34{ }^{\circ} \mathrm{C}$. Para lograrlo, se debe colocar el servocontrol de la servocuna o incubadora en $35^{\circ} \mathrm{C}$.

- Si la temperatura rectal es inferior a $33{ }^{\circ} \mathrm{C}$, se debe cubrir al bebé y poner en marcha los mecanismos para aumentarla (encender la incubadora, etc.).

- Si la temperatura rectal cae por debajo de $32^{\circ} \mathrm{C}$, se debe llamar al centro de mayor complejidad para obtener instrucciones adicionales.

En todo centro de atención que no cuente con UCIN, una vez determinada la presencia de asfixia perinatal, debe realizarse la estabilización del paciente y asegurar, al menos, una vía venosa periférica o central para mantener una glucemia óptima y el aporte de volumen durante la reanimación y / o después de los primeros minutos de vida. Se debe mantener permanentemente apagada la servocuna. La temperatura rectal objetivo de traslado es de entre $33{ }^{\circ} \mathrm{C}$ y $34{ }^{\circ} \mathrm{C}$. El traslado debe realizarse en incubadora con el servocontrol en $35^{\circ} \mathrm{C}$. Se debe mantener un control estricto de temperatura, idealmente con monitor, y, de no ser posible, se debe controlar la temperatura rectal, al menos, cada $15 \mathrm{~min}$. Para ello, se debe disponer de termómetros especiales que marquen temperatura menor de $35^{\circ} \mathrm{C} .{ }^{23-25}$

\section{Manejo del paciente durante la hipotermia}

El tratamiento con hipotermia moderada debe llevarse a cabo en unidades de Neonatología de nivel terciario ${ }^{1}$ con capacidad de atención multidisciplinaria y con protocolos estandarizados. $^{3}$

\section{Medidas generales}

La temperatura central debe vigilarse mediante monitorización con sonda rectal introducida de 5 a $6 \mathrm{~cm}$ en el recto o esofágica para evitar episodios de hipertermia o hipotermia excesiva $\left(<33^{\circ} \mathrm{C}\right)$. Se recomienda obtener un valor de temperatura no invasiva (axilar o hipocondrio derecho) que proporcione seguridad en caso de mal funcionamiento de la sonda rectal o esofágica. Una temperatura esofágica de $33,5^{\circ} \mathrm{C}$ se corresponde con una temperatura superficial de $31,5^{\circ} \mathrm{C}-32{ }^{\circ} \mathrm{C}$. Todas las decisiones clínicas deben ser tomadas sobre la base de la temperatura central. ${ }^{1,26}$

La hipotermia debe ser iniciada dentro de las $6 \mathrm{~h}$ del nacimiento a $33,5^{\circ} \mathrm{C}$ si es corporal total $\mathrm{y}$, si es selectiva, el rango de temperatura central es de $34{ }^{\circ} \mathrm{C}-35^{\circ} \mathrm{C}$ y la duración debe ser de $72 \mathrm{~h}$. Prolongar o profundizar la hipotermia no ha mostrado beneficios e, incluso, puede ser perjudicial. ${ }^{27}$

Manejo respiratorio: La necesidad de asistencia respiratoria mecánica (ARM) será evaluada en cada paciente según su estado clínico y, en caso de requerir sedoanalgesia, según su tolerancia. Es importante recordar que la hiperoxia incrementa el daño oxidativo luego de la asfixia, por lo que se debe controlar el nivel de oxígeno requerido.

La hipertensión pulmonar durante el tratamiento con hipotermia debe ser manejada en forma habitual. El uso de hipotermia terapéutica no modifica las indicaciones de óxido nítrico inhalatorio $(\mathrm{ONi}) \mathrm{u}$ oxigenación por membrana extracorpórea (Extracorporeal Membrane Oxygenation; ECMO, por sus siglas en inglés) en estos pacientes.

Se debe recordar calentar y humidificar el aire inspirado a igual temperatura que cualquier paciente ventilado $\left(36^{\circ} \mathrm{C}\right)$.

Los niveles de $\mathrm{CO}_{2}$ no deben ser menores de $35 \mathrm{mmHg}$ si el paciente está ventilado, ya que la hipocapnia en las primeras horas de vida se asoció a mayor mortalidad y discapacidad a los 18-22 meses. ${ }^{10}$

Es importante expresar algunas consideraciones técnicas: la presión parcial de $\mathrm{CO}_{2}\left(\mathrm{pCO}_{2}\right)$ disminuye un $4 \%$ por cada grado de reducción en la temperatura del paciente, por lo que existe más $\mathrm{CO}_{2}$ disuelto en la sangre. Los analizadores de gases realizan la medición de estos suponiendo una temperatura de $37^{\circ} \mathrm{C}$. En hipotermia, se debe cambiar la temperatura de la muestra en el analizador. Una muestra analizada a $37^{\circ} \mathrm{C}$ con valores de $\mathrm{pCO}_{2}$ de $36-44 \mathrm{mmHg}$ dará valores de $\mathrm{pCO}_{2}$ de $31-36 \mathrm{mmHg}$ a $33,5{ }^{\circ} \mathrm{C} .{ }^{26} \mathrm{Se}$ recomienda avisar al laboratorio que analiza la muestra la temperatura del paciente.

Manejo hemodinámico: Desde el aspecto cardiovascular, durante el tratamiento con hipotermia, el consumo de oxígeno del 
miocardio y el gasto cardíaco se reducen. Puede presentarse bradicardia sinusal sin compromiso hemodinámico; en raros casos, se reportaron arritmias graves. ${ }^{26}$

Es importante evaluar el compromiso de la contractilidad miocárdica, que puede determinar la necesidad de apoyo con inotrópicos. Para ello, debe efectuarse una evaluación cardiológica con ecocardiograma que permita estimar el grado de disfunción miocárdica, evaluar signos de hipertensión pulmonar y descartar cardiopatía estructural. Si la contractilidad miocárdica es pobre, se prefiere la administración de dobutamina; si el bebé está hipotenso, no tiene mala contractilidad, no está hipovolémico y aparece clínicamente vasodilatado, puede ser preferible el uso de dopamina o adrenalina. Se debe recordar que el $\mathrm{RN}$ aumenta el volumen minuto cardíaco mediante el aumento de la frecuencia cardíaca y, dadas las características del miocardio, tolera mal las expansiones; por lo tanto, si estas son necesarias, deben ser realizadas cuidadosamente.

El compromiso hemodinámico y la disminución de la capacidad de autorregulación del flujo sanguíneo cerebral hacen especialmente importante el mantenimiento de una presión arterial media en el rango normal, por lo que debe ser controlada de modo estrecho.

El valor del ácido láctico es una herramienta útil para evaluar la perfusión tisular. Si bien la hipotermia desplaza la curva de disociación de la hemoglobina a la izquierda y reduce la entrega de oxígeno a los tejidos, la perfusión disminuye proporcionalmente a la demanda, por lo que el metabolismo anaerobio no se ve incrementado. Es por ello por lo que la elevación del lactato depende solo de la gravedad de la asfixia y no del tratamiento con hipotermia. ${ }^{26}$

La hipoglucemia en estos pacientes ha mostrado tener un efecto deletéreo en cuanto a la acentuación del daño cerebral, por lo cual la glucemia plasmática debe ser evaluada a fin de mantenerla en el rango normal: entre 70 y $120 \mathrm{mg} / \mathrm{dl}$.

Luego de una agresión hipóxico-isquémica, existe riesgo de hipoglucemia. Sin embargo, durante la fase de mantenimiento de la hipotermia, probablemente por una disminución del metabolismo, habrá una tendencia a la hiperglucemia. Luego, durante la etapa de recalentamiento, vuelve a existir riesgo de hipoglucemia. Por ello, es necesario controlar las cifras de glucemia antes, durante y después del tratamiento con hipotermia.
No se recomienda la utilización de insulina en caso de hiperglucemia durante la fase de mantenimiento. ${ }^{26}$

Desde el aspecto hidroelectrolítico, se recomienda la restricción hídrica con aportes entre 40 y $50 \mathrm{ml} / \mathrm{kg} /$ día. El balance de ingresos y egresos debe ser estricto por la posible afectación renal y la probabilidad de presentar síndrome de secreción inadecuada de hormona antidiurética (SIHAD) con oligoanuria, sobrecarga hídrica e hiponatremia. Es necesario el control de las cifras de electrolitos, calcio y magnesio. La alteración de estos puede agravar o potenciar las crisis convulsivas. El objetivo es mantener unas cifras de calcio total $>8,5 \mathrm{mg} / \mathrm{dl}$ (calcio iónico $>1 \mathrm{mmol} / \mathrm{l}$ ), magnesio $>1,9 \mathrm{mg} / \mathrm{dl}$, sodio entre 135 y $145 \mathrm{mEq} / \mathrm{L}$ y potasio entre 3,5 y 4,5 mEq/L. Se debe tener especial cuidado con los aportes de potasio, ya que la hipotermia produce cambios en el potasio intracelular y puede producir una hiperpotasemia de rebote durante la fase de recalentamiento. ${ }^{1}$

Respecto de los accesos vasculares, se sugiere disponer de un catéter umbilical venoso y arterial. De no ser posible, se deben considerar vías periféricas. ${ }^{1}$ Es importante colocar los catéteres antes de iniciar la hipotermia, debido a que la vasoconstricción por ella puede producir mayor dificultad técnica. ${ }^{3}$

La evaluación neurológica debe ser realizada inicialmente por el neonatólogo para determinar el grado de encefalopatía y decidir el inicio de la hipotermia. Con posterioridad, el paciente deberá ser evaluado por un neurólogo infantil. Si se dispone de un monitor de función cerebral, se recomienda su uso desde el ingreso del niño para monitorizar la actividad cerebral durante las distintas fases de la hipotermia. El uso de fenobarbital profiláctico es controvertido y no se recomienda de rutina.

Los $R N$ con asfixia perinatal pueden presentar exámenes de coagulación alterados. Esto puede deberse a disfunción plaquetaria, disminución del número de plaquetas (durante la hipotermia, puede disminuir hasta un $40 \%$ el recuento plaquetario); además, puede presentarse prolongación del tiempo de protrombina y el de tromboplastina parcial activada. En caso de que la coagulación o el número de plaquetas se encuentre alterado, puede ser necesario realizar 
su corrección mediante transfusión de plasma y/o plaquetas.

Tanto la monitorización como los controles clínicos y de laboratorio iniciales están orientados a establecer el grado de encefalopatía y de afectación multiorgánica asociada (hepática, miocárdica, renal, respiratoria, etc.) (Tabla 2).

\section{Realización de controles de laboratorio}

La Tabla 3 es una guía de las determinaciones que deben ser realizadas. Se debe recordar que, si hay alteraciones del EAB que requieren ajustes del respirador, los controles deberán ser más frecuentes, de igual manera con las alteraciones hidroelectrolíticas.

\section{TABLA 2. Resumen por los aspectos que se deben realizar}

\section{Respiratorio}

Se debe intubar y ventilar en los siguientes casos:

- Hay respiraciones espontáneas ineficaces.

- Desaturación a pesar de la administración de oxígeno $\left(\mathrm{O}_{2}\right)$ a flujo libre.

- Insuficiencia respiratoria en los gases sanguíneos.

- Crisis convulsivas tempranas que interfieran con la respiración.

- La administración de $\mathrm{O}_{2}$ mediante una cánula nasal probablemente sea una mejor estrategia que el uso de halo cefálico con el objetivo de evitar la hipertermia.

- Se debe monitorear la saturación de oxígeno continua, con un objetivo de saturación preductal de $90 \%$ a $95 \%$.

- Se deben realizar ajustes del respirador con los objetivos trazados de límites de presión parcial de dióxido de carbono $\left(\mathrm{pCO}_{2}\right)$ y presión parcial de oxígeno $\left(\mathrm{PO}_{2}\right)$.

- Se debe realizar estado ácido-base (EAB) de acuerdo con las necesidades del paciente.

\section{Cardiovascular}

- Se debe realizar la monitorización cardiorrespiratoria continua con monitor multiparamétrico (frecuencia cardíaca, frecuencia respiratoria, presión arterial).

- Se debe realizar ecocardiografía.

- La hipotensión es una consecuencia común de la isquemia miocárdica. Solo si se sospecha hipovolemia, se deben dar 10-20 ml/kg de solución fisiológica.

- Se debe considerar administrar inotrópicos.

- Se debe recordar que la hipoxia produce alteración de las enzimas miocárdicas, por lo tanto, es aconsejable solicitar su dosaje al ingresar y posteriormente. Se deben monitorear los valores de ácido láctico.

\section{Metabólico}

- Se deben realizar controles de glucemia y EAB.

- Se debe solicitar un hepatograma.

- Se debe asegurar el aporte de un flujo de glucosa suficiente para prevenir la hipoglucemia y monitorear los niveles de glucosa para evitar la hiperglucemia.

- Se deben evitar las correcciones con bicarbonato teniendo en cuenta las causas de acidosis metabólicas para tratarlas.

\section{Líquidos y electrolitos}

- Se debe colocar una sonda nasogástrica (SNG) abierta.

- Se deben restringir los fluidos a 40-50 ml/ kg/ día. La restricción de líquidos es esencial, debido al riesgo de secreción inapropiada de hormona antidiurética e insuficiencia renal.

- Se debe evaluar el ritmo diurético.

- Se debe realizar el control de electrolitos, calcio, magnesio, urea y creatinina.

\section{Nutricional}

- Se debe iniciar nutrición parenteral total (NPT) desde el primer día de vida y considerar los beneficios de administrar calostro.

\section{Infectológico}

- Si existe la posibilidad de sepsis, se deben tomar cultivos de sangre y comenzar un tratamiento antibiótico con ampicilina y gentamicina. Se debe realizar la curva de reacción en cadena de la polimerasa (polymerase chain reaction; PCR, por sus siglas en inglés) cuantitativa y, si los cultivos son negativos, suspender el antibiótico precozmente.

\section{Neurológico}

- Si se dispone del equipo, se debe realizar un electroencefalograma de amplitud integrada (EEGa).

- Se debe realizar una ecografía cerebral para descartar malformaciones del sistema nervioso central (SNC) o hemorragias.

\section{Hematológico}

- Se debe monitorear hematocrito, recuento de plaquetas, tiempo de protrombina (TP), tiempo de tromboplastina parcial activada (KPTT) y fibrinógeno, y realizar las correcciones necesarias. 


\section{Pasos iniciales del tratamiento}

- La preparación del equipo de hipotermia demora, aproximadamente, 30-40 minutos, por lo que es importante considerar este punto ante un paciente eventual. ${ }^{26}$

- Si se dispone de un monitor, se debe iniciar la monitorización continua de la función cerebral mediante electroencefalograma de amplitud integrada (EEGa).

- Se debe dar información a los padres acerca del tratamiento que va a recibir el niño.

\section{FASES}

El tratamiento con hipotermia se realiza en 3 fases. Tiene como objetivo alcanzar una temperatura determinada, sostenerla en ese nivel durante $72 \mathrm{~h}$ y permitir el recalentamiento en forma lenta.

\section{Fase de enfriamiento}

En estudios experimentales, se ha demostrado que, cuanto más precozmente se realice el enfriamiento, mayor es la eficacia del tratamiento. ${ }^{28}$

El objetivo es reducir la temperatura central a $33{ }^{\circ} \mathrm{C}-34{ }^{\circ} \mathrm{C}$ en la hipotermia corporal total y a $34{ }^{\circ} \mathrm{C}-35^{\circ} \mathrm{C}$ en la selectiva de la cabeza en $30-$ 40 minutos. El enfriamiento debe ser más lento en niños con necesidades de oxígeno mayores de $50 \%$ o si aumentan más de $30 \%$ sobre el valor inicial debido al riesgo de hipertensión pulmonar. ${ }^{26}$

TABLA 3. Controles de laboratorio

\begin{tabular}{lccc}
\hline & Al ingresar & $\mathbf{1 2 ~ h}$ & $\mathbf{2 4} \mathbf{~ h}$ \\
\hline EAB & $\mathrm{X}$ & $\mathrm{X}$ & $\mathrm{X}$ \\
Ác. láctico & $\mathrm{X}$ & $\mathrm{X}$ & $\mathrm{X}$ \\
Hemograma & $\mathrm{X}$ & & $\mathrm{X}$ \\
Rto. de plaquetas & $\mathrm{X}$ & & $\mathrm{X}$ \\
Coagulograma & $\mathrm{X}$ & & $\mathrm{X}$ \\
Glucemia & $\mathrm{X}$ & Cada $6 \mathrm{~h}$. & $\mathrm{X}$ \\
Ionograma & & $\mathrm{X}$ & $\mathrm{X}$ \\
Calcio & & $\mathrm{X}$ & $\mathrm{X}$ \\
GOT/GPT & $\mathrm{X}$ & & $\mathrm{X}$ \\
Amonio & & & $\mathrm{X}$ \\
Fósforo/magnesio & & & $\mathrm{X}$ \\
Urea & $\mathrm{X}$ & & $\mathrm{X}$ \\
Creatinina & $\mathrm{X}$ & & $\mathrm{X}$ \\
Enzimas cardíacas & $\mathrm{X}$ & & \\
Hemocultivos & $\mathrm{X}$ & & \\
\hline
\end{tabular}

EAB: estado ácido-base; GOT: transaminasa glutámico oxalacética; GPT: transaminasa glutámico-pirúvica.

\section{Fase de mantenimiento}

En esta etapa, el objetivo es mantener la temperatura deseada durante $72 \mathrm{~h}$ en forma estable. Puede ser necesario prolongar su duración según la respuesta del paciente al recalentamiento (presencia de convulsiones).

En ninguna circunstancia, el tratamiento con hipotermia debe suspenderse por la mejoría del estado neurológico del RN (ni clínico ni del registro del EEGa).

Es importante mantener una adecuada monitorización para el control de los signos vitales, EEGa, de ingresos y egresos, de laboratorio y confort del niño. En este sentido, los fármacos más utilizados para la sedación son los opiáceos, en especial, la morfina. El metabolismo hepático se encuentra reducido, por lo que deben utilizarse dosis menores y titular su respuesta. No se aconseja el empleo de relajantes musculares, dado que no ofrecen beneficios y pueden enmascarar crisis convulsivas clínicas. ${ }^{29}$

\section{Fase de recalentamiento}

El objetivo de esta etapa es regresar al niño a la temperatura normal en forma lenta y controlada. Se estima un incremento de $0,2{ }^{\circ} \mathrm{C}-0,5^{\circ} \mathrm{C}$ por hora.

Durante este período, debe controlarse la presión arterial, dado que puede producirse vasodilatación periférica, y también el nivel de potasio en sangre, ya que los cambios en el potasio intracelular asociados al recalentamiento pueden presentarse con hiperkalemia. Uno de los síntomas que deben controlarse con especial cuidado es la presencia de convulsiones. Al incrementar la temperatura, aumenta el metabolismo cerebral y, con este, el riesgo de convulsiones. De contar con monitor de EEGa, su uso es fundamental para detectar la presencia de convulsiones subclínicas. Puede ser necesario detener o enlentecer la etapa de recalentamiento. ${ }^{30}$

Luego de alcanzar una temperatura central de $36,5^{\circ} \mathrm{C}-37^{\circ} \mathrm{C}$, puede retirarse la sonda rectal o esofágica y continuar el control de la temperatura con mediciones periféricas.

\section{MODOS DE TRATAMIENTO}

Hipotermia pasiva: se refiere al descenso de la temperatura corporal que se logra al suspender todas las medidas de calentamiento al RN. Solo posee utilidad en los centros derivantes a la espera del transporte y durante este para optimizar el tiempo de inicio del tratamiento. ${ }^{31}$ 
Hipotermia activa: es el modo de elección. Se logra mediante la utilización de equipos de enfriamiento. Puede ser de dos formas: hipotermia corporal total o selectiva de la cabeza.

Hipotermia corporal total: se utilizan equipos que constan de una manta o colchón por los que circula agua a diferentes temperaturas, que permite mantener al paciente en el rango de temperatura deseada. Algunos de estos aparatos disponen de servocontrol, lo que permite mantener la temperatura más estable y realizar modificaciones de la temperatura del agua circulante en forma automática. Poseen la ventaja de mantener un adecuado acceso a la cabeza del niño para la colocación del EEGa y la evaluación ecográfica.

Hipotermia cerebral selectiva: se utiliza un equipo diseñado para el enfriamiento cerebral. Posee un gorro especial por el que circula agua que permite mantener la temperatura rectal a $34{ }^{\circ} \mathrm{C}-35{ }^{\circ} \mathrm{C}$. Durante el tratamiento con hipotermia, el paciente permanece en una cuna servocontrolada bajo calor radiante. De este modo, se optimiza la hipotermia en la corteza cerebral, se alcanzan temperaturas en el cuero cabelludo de $23^{\circ} \mathrm{C}-28^{\circ} \mathrm{C}$ y se mantiene una temperatura central en $34^{\circ} \mathrm{C}-35^{\circ} \mathrm{C} .{ }^{32}$

\section{Tratamiento farmacológico}

El tratamiento antibiótico en pacientes con sospecha de sepsis es ampicilina y gentamicina. Excepto en los casos en que exista insuficiencia renal asociada al evento hipóxico-isquémico, la eliminación renal no se encuentra alterada. Con respecto al metabolismo hepático, la hipotermia lo disminuye. La morfina presenta un aclaramiento lento, por lo que se recomienda disminuir su dosis.

Respecto del tratamiento de las convulsiones, cada centro debe utilizar su protocolo de tratamiento habitual. Es importante realizar el monitoreo de los niveles sanguíneos y el ajuste de las dosis de medicamentos, como el fenobarbital.

\section{Monitoreo de la función cerebral Monitoreo por electroencefalograma de amplitud integrada}

El equipo de EEGa o monitor de función cerebral proporciona información sobre la actividad eléctrica global del cerebro mediante el registro de un canal único o doble, comprimido y filtrado. ${ }^{33}$ Esto significa que, a diferencia del

FIGURA 1. Registro normal: trazado superior de electroencefalograma de amplitud integrada con margen superior $>10 \mathrm{mV}$ e inferior $>5 \mathrm{mV}$.

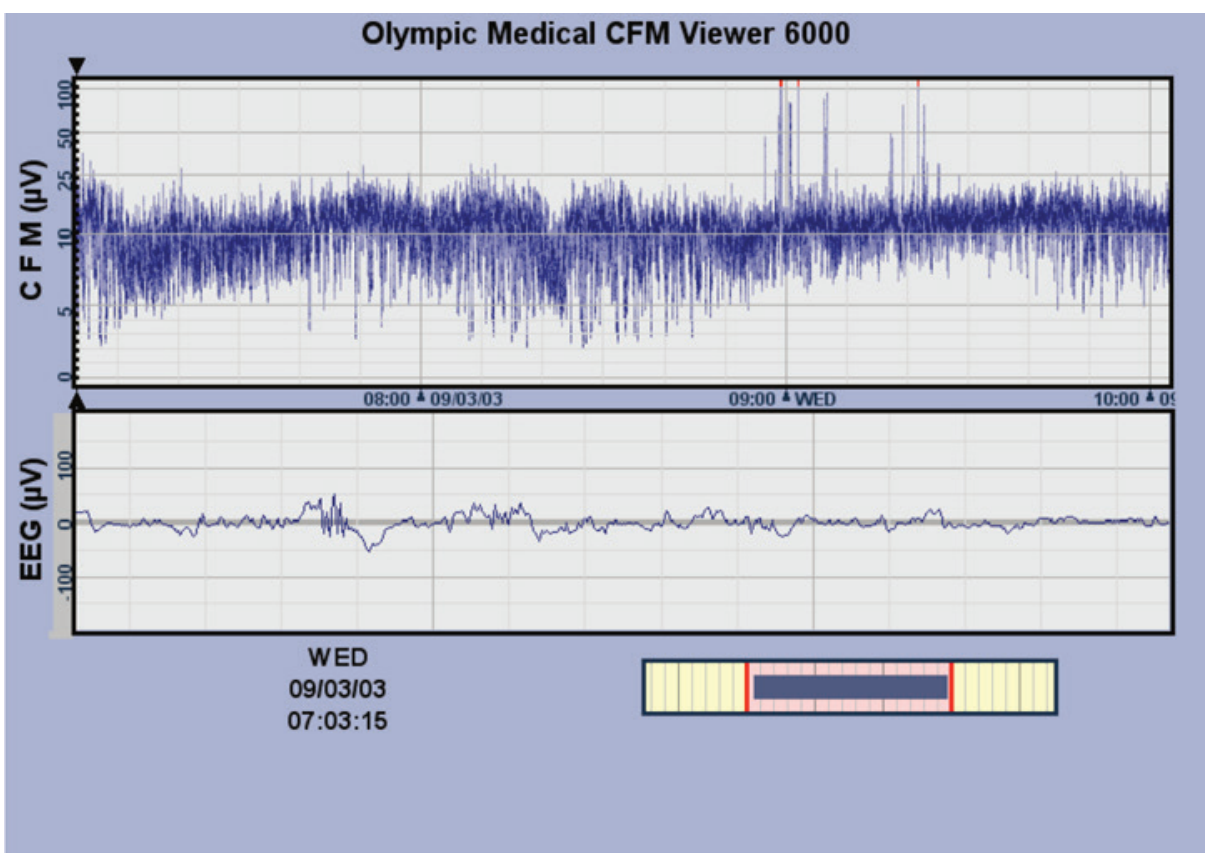

EEG: electroencefalograma; CFM: monitor de función cerebral. 
electroencefalograma convencional modificado para neonatos, que utiliza 9 electrodos, el EEGa usa de 2 a 4 electrodos de registro (según sea de 1 o 2 canales) y permite una evaluación en tiempo real más simple, menos costosa, reproducible $\mathrm{y}$, relativamente, de fácil interpretación para el equipo de salud que atiende al neonato. Sin embargo, el electroencefalograma convencional sigue siendo el gold standard para la evaluación de las convulsiones (en el mejor de los casos, el EEGa solo detecta el $80 \%$ de ellas). Para entender cómo registra el EEGa, se debe pensar que las ondas del electroencefalograma se comprimen en una línea que resume 15 segundos de registro convencional. Por lo tanto, el trazado superior muestra en forma permanente 3 horas de actividad cerebral continua (Figura 1). El trazado inferior puede mostrar, en forma alternativa, dos registros diferentes. En primer lugar, la impedancia que interfiere en la captación de la actividad eléctrica $\mathrm{y}$, en segundo lugar, el electroencefalograma convencional en tiempo real. La impedancia debe ser menor de 10 para que la evaluación sea confiable (Figura 2).

La utilidad del EEGa es proporcionar información adicional sobre la gravedad de la encefalopatía y la evaluación de la actividad convulsiva. ${ }^{33}$ Por lo tanto, puede contribuir a la selección de los candidatos a hipotermia cuando existen dudas sobre su indicación.
Cuando este equipo no se encuentra disponible, el inicio de la hipotermia pasiva y el traslado a un centro de nivel III con programa de hipotermia están bien fundados si existen antecedentes de asfixia perinatal junto con la presencia de disfunción neurológica significativa.

El tiempo para la recuperación de un trazado normal de un registro de EEGa parece ser un buen predictor de resultado en los RN con EHI tratados con hipotermia. La hipotermia modifica el valor pronóstico del EEGa temprano (3-6 h). La normalización del EEGa en los pacientes tratados con hipotermia se produce en forma más tardía $(\sim 48 \mathrm{~h})$ que en los pacientes con normotermia $(\sim 24 \mathrm{~h})$. El tiempo transcurrido hasta la normalización del trazado es mejor predictor que el tiempo requerido en retomar el ciclo sueño-vigilia. No recuperar este patrón es también predictor de mal pronóstico. ${ }^{34}$

Un EEGa normal no contraindica el inicio de la hipotermia si el neonato presenta encefalopatía moderada o grave.

¿Cómo se evalúan los registros del electroencefalograma de amplitud integrada?

En el trazado superior, se evalúan los márgenes superior e inferior de este (no se deben tener en cuenta las líneas que sobresalen de la sombra global). Para que sea normal, el primero debe estar por encima de $10 \mathrm{mV}$, y el segundo debe ser mayor de $5 \mathrm{mV}$, como se observa en la Figura 1.

FIGURA 2. Trazado de electroencefalograma de amplitud integrada normal: la flecha indica que la línea de la impendancia es cercana a "0", por lo cual se considera que el registro del trazado superior es confiable. Los cambios del ancho del trazado superior revelan los distintos ciclos de sueño normales.

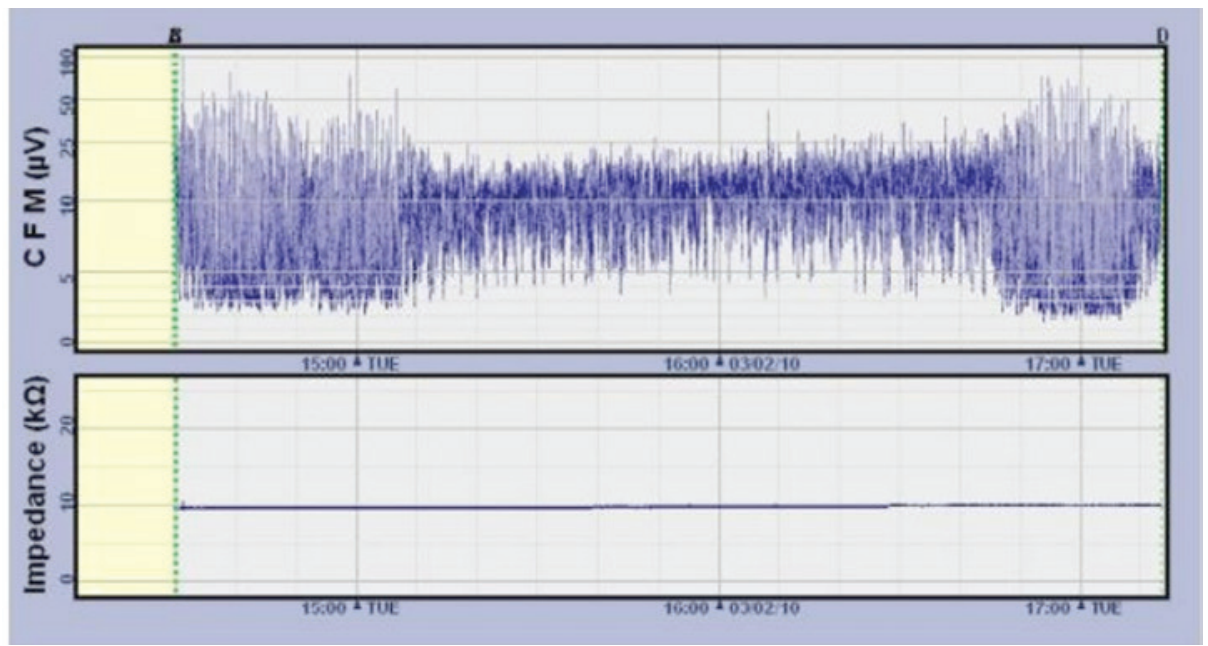


En la Figura 3, se ven diferentes grados de gravedad de la EHI evaluada a través del EEGa.

\section{Actividad convulsiva}

La actividad convulsiva puede detectarse cuando el trazado superior se eleva y se estrecha. Para corroborarlo, se debe verificar que el trazado inferior tenga una impedancia adecuada y que el electroencefalograma revele ondas anormales (véase la Figura 4). Cuando estos cambios se vuelven repetitivos, adquieren el patrón de "diente de pescado" o "en serrucho" y esto refleja un "estatus convulsivo" (Figura 4).

\section{Eventos adversos}

Los eventos adversos más comunes de la hipotermia moderada son la presencia de bradicardia sinusal y trombocitopenia (recuento

FIGURA 3. Gravedad de la encefalopatía hipóxico-isquémica evaluada a través del electroencefalograma de amplitud integrada:
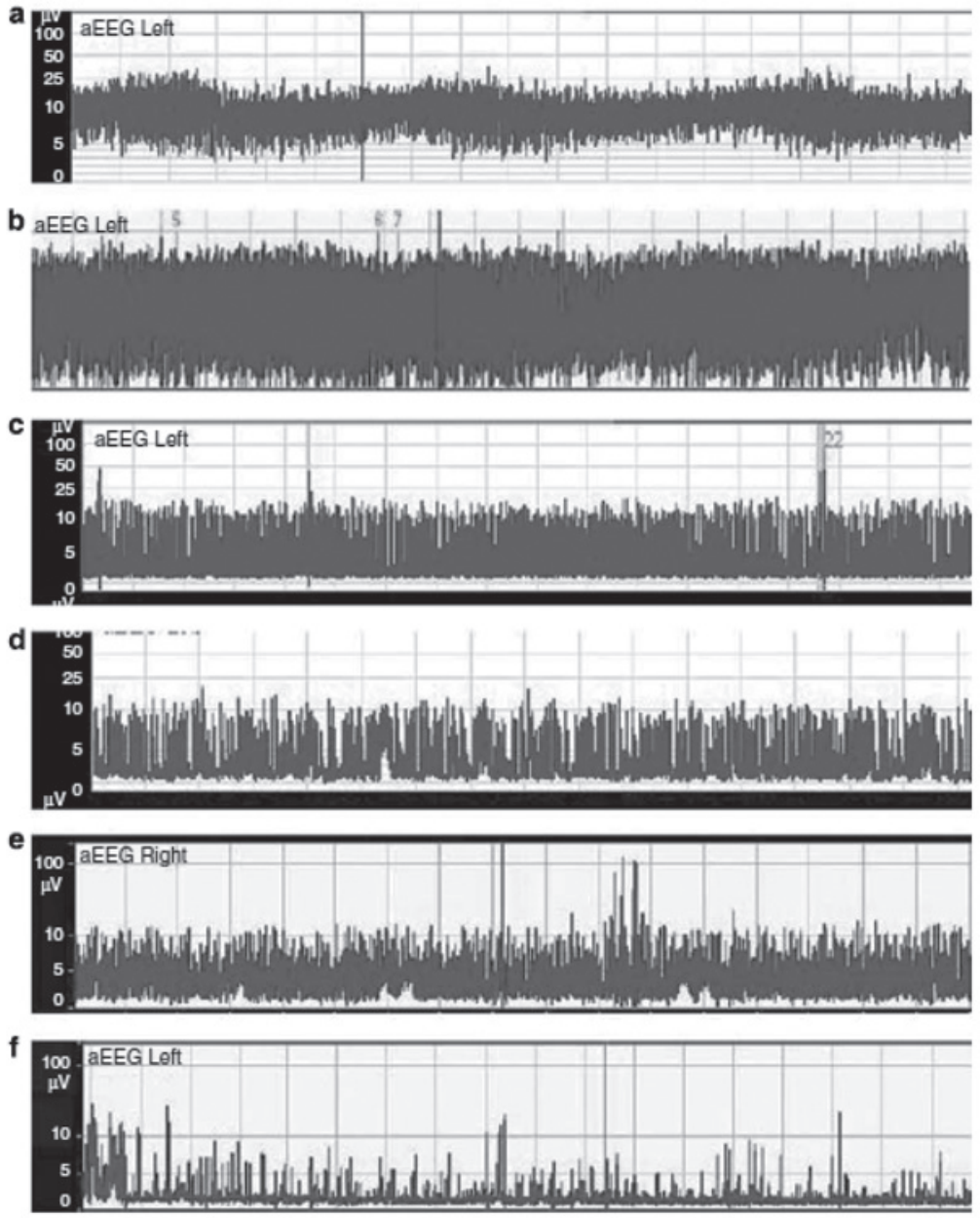

a. Patrón continuo normal: con margen superior $>10 \mathrm{mV}$ e inferior $>5 \mathrm{mV}$. Las variaciones en el ancho del trazado reflejan los ciclos de sueño normales.

b. Patrón discontinuo: con margen superior $>10 \mathrm{mV}$, pero con margen inferior $<5 \mathrm{mV}$. Según el contexto clínico, puede ser normal (inmadurez, anticonvulsivantes o sedantes) o moderadamente anormal.

c. Patrón Burst Suppression o descargas con supresión: con margen superior $<10 \mathrm{mV}$ e inferior $<5 \mathrm{mV}$ con descargas múltiples (> 100/hora). Desorganizado, gravemente anormal.

d. Patrón Burst Suppression o descargas con supresión: con margen superior $<10 \mathrm{mV}$ e inferior $<5 \mathrm{mV}$ con descargas $<100 /$ hora. Gravemente anormal.

e. Patrón de bajo voltaje continuo: con margen superior e inferior muy por debajo de $10 \mathrm{mV}$ y $5 \mathrm{mV}$, respectivamente. Picos ocasionales. Gravemente anormal.

f. Trazado "plano" o isoeléctrico: ambos márgenes por debajo de $5 \mathrm{mV}$. Prominentes picos debido a movimientos del paciente. Anormal, gravedad extrema. 
de plaquetas $\left.<150000 / \mathrm{mm}^{3}\right) .{ }^{35}$ Con menor frecuencia, se han observado enrojecimiento o endurecimiento de la piel y necrosis grasa subcutánea. ${ }^{36}$

\section{Estudio del sistema nervioso central con imágenes}

La resonancia magnética ( $\mathrm{RMN}$ ) cerebral es el estudio de elección para evaluar la lesión hipóxico-isquémica del $\mathrm{RN}$ y se recomienda su realización en el período neonatal.

Las áreas cerebrales lesionadas durante la hipoxia-isquemia dependerán de la edad gestacional del niño y de la gravedad y duración del evento hipóxico-isquémico. En los niños nacidos a término que sufren EHI, hay cuatro patrones principales de lesión, de los cuales los 2 primeros son los más frecuentemente observados: ${ }^{37}$

- Lesión central de ganglios basales y tálamo (LGT)

- Lesión de áreas limítrofes

- Lesión difusa (cerebro blanco)

- Lesiones periventriculares

También pueden encontrarse lesiones focales isquémicas o hemorrágicas y hemorragias extraaxiales como patologías asociadas.

La LGT, el patrón más frecuente, se asocia a una hipoxia-isquemia profunda pero breve. Las lesiones se observan en el tálamo ventrolateral y el putamen posterior, el brazo posterior de la cápsula interna y la corteza perirrolándica, ya que son las áreas que se están mielinizando a la edad de término y, por ende, las más sensibles a la hipoxia-isquemia. Si el evento se prolonga, pueden lesionarse los hipocampos, el tronco cerebral y el cerebelo. La pérdida de visualización del brazo posterior de la cápsula interna y de la diferenciación entre la corteza y la sustancia blanca son signos precoces de lesión hipóxicoisquémica.

Las lesiones en áreas limítrofes se asocian a eventos isquémicos menos graves pero más prolongados. La lesión se observa en la sustancia blanca subcortical parasagital (límite entre la irrigación de la arteria cerebral media y anterior y entre la media y posterior) y, en casos graves, también compromete la corteza adyacente.

La elección de las secuencias por realizar es fundamental para un resultado óptimo. La técnica de difusión y ADC (apparent diffusion coefficient) tienen mejor sensibilidad para la observación de lesiones isquémicas en comparación con las secuencias convencionales (T1 y T2), pero son positivos solo hasta alrededor del día 10 posterior a la isquemia. A partir de ese momento, las lesiones deberán evaluarse en las secuencias convencionales, lo que, en ocasiones, requiere gran experiencia del observador. En las primeras $48 \mathrm{~h}$ luego del evento hipóxico-isquémico, la secuencia de difusión puede ser negativa y, en ese caso, la única anomalía visible será la presencia de ácido láctico en la espectroscopia.

Otras secuencias de utilidad son el gradiente de eco (GRE) o las imágenes por susceptibilidad, ambas sensibles para la visualización de productos de degradación de la hemoglobina $\mathrm{y}$, por lo tanto, útiles para observar lesiones hemorrágicas que pasarían desapercibidas en las secuencias convencionales.

La RMN cerebral debería realizarse, idealmente, a partir del día 4 o 5 de vida, ya habiendo finalizado el tratamiento de hipotermia y con el niño estable y trasladable, y antes del día 10 de vida para obtener imágenes visibles en la secuencia de difusión. Si esta se realiza

FIGURA 4. Estatus convulsivo en el electroencefalograma de amplitud integrada: en el trazado superior, se observan múltiples estrechamientos y elevaciones, que revelan convulsiones repetidas. En el trazado inferior, se observa el electroencefalograma convencional con las formas típicas de actividad convulsiva (compárese con la Figura 1).

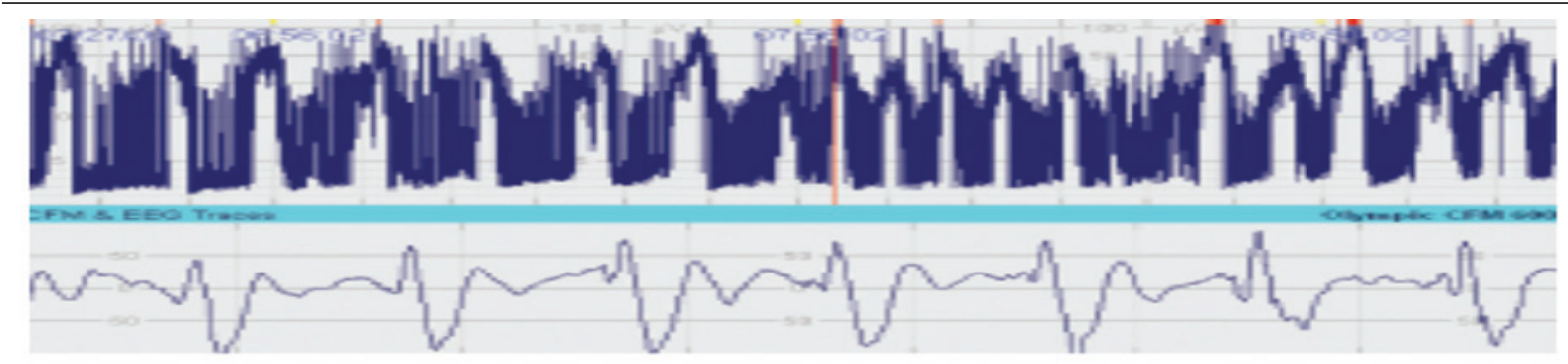


antes de ingresar en hipotermia, durante las primeras horas de vida, es fundamental hacer espectroscopia con voxel a nivel del tálamo capsular. Los estudios realizados después del día 10 o 14 de vida mostrarán las lesiones ya establecidas en las secuencias convencionales. La RMN puede realizarse mediante inducción del sueño con hidrato de cloral para evitar la anestesia general, colocando al niño en forma confortable y contenido para evitar movimientos y con protección auricular para disminuir la incomodidad del ruido del resonador. ${ }^{38}$

La tomografía computarizada ofrece menor definición y alta carga de radiación, por lo que su uso queda restringido a casos de sospecha de una patología de resolución neuroquirúrgica urgente.

La ecografía cerebral es inocua y altamente accesible, útil para tener una primera aproximación en los casos de niños inestables que no puedan ser trasladados, pero su información es limitada en casos de EHI.

Es fundamental realizar una RMN en un paciente con EHI grave al finalizar el tratamiento con hipotermia para documentar la gravedad de las lesiones. El resultado de este estudio, junto con la clínica y los estudios de electroencefalogramas, guiará la conducta posterior en relación con el manejo del paciente y la información a la familia; ej.: si el daño del SNC es grave, es obligatorio considerar la adecuación de esfuerzo terapéutico, consensuar cuidados de confort y evitar tratamientos (traqueotomía, gastrostomía) fútiles.

\section{Potenciales auditivos evocados automatizados}

Se debería realizar, al menos, un estudio antes del egreso del paciente de la Unidad.

\section{Nuevos tratamientos coadyuvantes}

Si bien la hipotermia demostró los beneficios antes descritos en los pacientes asfixiados, actualmente, hay varias líneas de investigación para mejorar los resultados en ellos.

Estos estudios asocian la hipotermia con los siguientes:

- Factor estimulante de la eritropoyesis

- Terapias con células madre

- Administración de gas xenón

- Administración de topiramato

\section{Seguimiento del paciente}

El alta del paciente con diagnóstico de EHI obliga a su inclusión en un programa de seguimiento multidisciplinario (pediatra, neurólogo, fisiatras de rehabilitación, psicólogos y servicio social) para su atención y la de su familia. Se debe recordar que las discapacidades tienen un impacto familiar y social, por lo tanto, es obligatorio pensar en un sostén, aun cuando las familias no manifiesten un requerimiento específico. Si el centro que realizó el tratamiento con hipotermia no cuenta con un equipo multidisciplinario para el seguimiento del paciente, debiera realizar la derivación oportuna al programar el alta.

\section{BIBLIOGRAFÍA}

1. Blackmon LR, Stark AR, the Committee on Fetus and Newborn, American Academy of Pediatrics. Hypothermia: A neuroprotective therapy for neonatal hypoxic-ischemic encephalopathy. Pediatrics 2006;117:942-8.

2. García-Alix A, Martínez-Biarge M, Diez J, Gayá F, Quero J. Encefalopatía neonatal hipóxico-isquémica: Incidencia y prevalencia en la primera década del siglo XXI. An Pediatr (Barc) 2009;71:319-26.

3. Hull J, Dodd KL. Falling incidence of hypoxic-ischaemic encephalopathy in term infants. Br J Obstet Gynaecol 1992; 99:386-91.

4. Gluckman PD, Wyatt JS, Azzopardi D, Ballard R, Edwards AD, Ferriero D M, et al. Selective head cooling with mild systemic hypothermia after neonatal encephalopathy: multicentre randomised trial. Lancet. 2005;365:663-70.

5. Shankaran S, Laptook AR, Ehrenkranz RA, Tyson JE, McDonaldSA, DonovanET, etal. Whole-body hypothermia for neonates with hypoxic-ischemic encephalopathy. New Engl J Med 2005;353:1574-84.

6. Eicher DJ, Wagner CL, Katikanemi LP. Moderate hypothermia in neonatal encephalopathy: safety outcomes. Pediatric Neurol 2005;32:18-24.

7. Azzopardi DV, Strohm B, Edwards AD, Dyet L, Halliday HL, Juszczak E, et al; TOBY Study Group. Moderate hypothermia to treat perinatal asphyxial encephalopathy. N Engl J Med 2009;361:1349-58.

8. Edwards AD, Azzopardi D V. Therapeutic hypothermia following perinatal asphyxia. Arch Dis Child Fetal Neonatal Ed 2006;91:F127-F131.

9. Schulzke S M, Rao S, Patole SK. A systematic review of cooling for neuroprotection in neonates with hypoxicischemic encephalopathy-are we there yet? BMC Pediatrics. 2007; 7:30. Disponible en: http//www.biomedcentral. com/1471-2431/7/30.

10. Shah PS, Ohlsson A, Perlman M. Hypothermia to treat neonatal hypoxic ischemic encephalopathy. Arch Pediatr Adolesc Med 2007;161:951-8.

11. JacobsS,HuntR, Tarnow-MordiW, InderT, DavisP. Cooling for newborns with hypoxic-ischemic encephalopathy. Cochrane Database Syst Rev 2007;(4): CD00311

12. Seetha Shankaran et al. Childhood Outcomes after Hypothermia for Neonatal Encephalopathy. N Engl J Med 2012;366:2085-92

13. Thoresen M, Wyatt JS. Keeping a cool head, posthypoxic hypothermia -an old idea revisited. Acta Paediatr 1997;86:1019-33.

14. Wyllie J, Perlman JM, Kattwinkel J, Wyckoff $\mathrm{MH}$, et al. Part 7: Neonatal resuscitation 2015 International Consensus on Cardiopulmonary Resuscitation and Emergency Cardiovascular Care Science with Treatment Recommendations. Resuscitation 2015; 95:e169-e201. 
15. Myra H. Wyckoff et al. Part 13: Neonatal Resuscitation 2015 American Heart Association Guidelines Update for Cardiopulmonary Resuscitation and Emergency Cardiovascular Care. Cardiopulmonary Resuscitation www.pediatrics.org/cgi/doi/10.1542/peds.2015-3373 G doi:10.1542/ peds.2015-3373G

16. Gunn AJ, Thoresen M. Hypothermic neuroprotection. Neuro Rx 2006;3:154-69.

17. Azzopardi D, Wyatt JS, Cady EB, Delpy DT, Baudin J, Stewart AL, et al. Prognosis of newborn infants with hypoxic-ischemic brain injury assessed by phosphorus magnetic resonance spectroscopy. Pediatr Res 1989;25:44551.

18. Hope PL, Cady EB, Tofts PS, Hamilton PA, Costelo A M, Delphy DT, et al. Cerebral energy metabolism studied with phosphorus NMR spectroscopy in normal and Barth asphyxiated infants. Lancet 1984;324:366-70.

19. Colbourne F, Corbett D, Zhao Z, Yang J, Buchan AM. Prolonged but delayed post-ischemic hypothermia: a long-term outcome study in the rat middle cerebral artery occlusion model. J Cereb Blood Flow Metab 2000;20:1702-8.

20. Gunn AJ, Benet L, Gunning MI, Gluckman PD, Gunn TR. Cerebral hypothermia is not neuroprotective when started after post ischemic seizures in fetal sheep. Pediatr Res 1999;46:274-80.

21. The American College of Obstetricians and Gynecologist. Neonatal Encephalopathy and Neurologic Outcome, Second Edition. Report of the American College of Obstetricians and Gynecologists. Task Force on Neonatal Encephalopathy. Obstetrics \& Gynecology 2014;123(4):896.

22. Vento M, Asensi M, Sastre J, Fernando García-Sala, Pallardó FV, Viña J. Resuscitation With Room Air Instead of $100 \%$ Oxygen Prevents Oxidative Stress in Moderately Asphyxiated Term Neonates. Pediatrics 2001;107(4):642-64.

23. Kendall G, Robertson N, Azzopardi D. Transport of infants referred for cooling treatment: cooling on retrieval clinical guidelines. UK TOBY -Cooling Register https://www. npeu.ox.ac.uk/downloads/files/toby register/TOBYRegister-Transport-Protocol.pdf. Accessed March 26, 2014.

24. Kendall G. Passive cooling for initiation of therapeutic hypothermia in neonatal encephalopathy. Arch Dis Child Fetal Neonatal Ed 2010:95; F408-f412.

25. O'Reilly D, Labrecque M, O'Melia M, Bacic J, Hansen A, Soul J. S. Passive cooling during transport of asphyxiated term newborns. J Perinatology 2013.Pág.435-40.
26. Blanco D et al. Comisión de estándares de la SEN. Neuroprotección con hipotermia en el recién nacido con encefalopatía hipóxico isquémica. Guía de estándares para su aplicación clínica. An Pediatr (Barc) 2011;75(5):341.e1341.e20

27. Shankaran S et al. NICHD. Effect of depth and duration of cooling on deaths in the NICU among neonates with hypoxic ischemic encephalopathy. JAMA 2014;312(24):2629-39.

28. Gunn AJ, Gunn TR, Gunning MI, Williams CE, Gluckman PD. Neuroprotection with prolonged head cooling started before post ischemic seizures in fetal sheep. Pediatrics 1998;102:1098-1106.

29. Novoa J, Milad M. Consenso sobre manejo integral del neonato con encefalopatía hipóxico isquémica. Rev Chil Pediatr 2012;83(5):492-501.

30. Polderman K. Induced hypothermia and fever control for prevention and treatment of neurological injuries. Lancet 2008:371:1955-69.

31. Jacobs SE, Morley CJ, Inder TE. Whole-body hypothermia for term and near-term newborns with hypoxic-ischemic encephalopathy: a randomized controlled trial. Arch Pediatr Adolesc Med 2011;165(8):692-700.

32. Ellis M, Manandhar D. Progress in perinatal asphyxia. Semin Neonatol 1999; 4:183-91.

33. Guidelines for Assessment and Initiation of Therapeutic Hypothermia(Cooling)TreatmentforBabies presenting with Moderate or Severe Hypoxic Ischaemic Encephalopathy. South Central Neonatal Steering Group for South Central North. June 8th 2011. Disponible en: www.networks.nhs. uk/...neonatal... / guidelines / Cooling

34. Thoresen M, Hellstrom-Westas L, Liu X, De Vries LS. Effect of hypothermia on amplitude-integrated electroencephalogram in infants with asphyxia. Pediatrics 2010;126:e131-e9.

35. Jacobs SE. Cooling for newborns with hypoxic ischaemic encephalopathy. Cochrane Database Syst Rev 2013 Jan 31;1: CD003311. Doi: 10.1002714651858.cd003311. PUB3

36. Committee on fetus and newborn. Hypothermia and neonatal encephalopathy. Pediatrics 2014;133:1146-50.

37. De Vries LS, Groenendaal F. Patterns of neonatal hypoxicischaemic brain injury. Neuroradiology 2010;52:555-66.

38. Rutherford M, Malamateniou C, McGuinness A, Allsop J, Martinez Biarge M, Counsell S. Magnetic resonance imaging in hypoxic-ischaemic encephalopathy. Early Human Development 2010;86:351-60. 